\title{
Vulvo-Perineal and Perianal Paget Disease. Radical Excision and Reconstruction with Singapore Flap
}

\author{
Theodoros Panoskaltsis ${ }^{1}$, Theofanis Panagiotis Arkoumanis ${ }^{2, *}$, Nikolaos Panagopoulos ${ }^{1}$, \\ Dimitrios Mastorakos ${ }^{2}$
}

\begin{abstract}
Extramammary Paget disease (EMPD) is an uncommon intraepithelial malignancy, affecting the vulvo-perineal and perianal region, occurring in 6.5\% of all Paget diseases. Usually, an underlying invasive adenocarcinoma denotes a more aggressive behaviour of the disease. We present the multidisciplinary approach in a 75-year old patient with this rare disease. The patient underwent a radical surgical excision and, subsequently, a Singapore flap was used for primary closure. The final histology confirmed the presence of a non-invasive Paget tumor, but a focus of high-grade invasive adenocarcinoma was noted in a perineal nodule. The histological margins were free of tumor. The patient did not undergo any adjuvant treatment because of severe chronic medical problems, although, eighteen months after treatment, she remains well, with no signs of recurrence. In conclusion, radical surgical excision, often necessitating reconstruction techniques, remains the gold standard of care and further adjuvant treatment should be individualised.
\end{abstract}

\section{KEYWORDS}

vulvo-perineal and perianal paget disease; Singapore flap

\section{AUTHOR AFFILIATIONS}

${ }^{1}$ Gynaecological Oncology Unit, 2nd Academic Department of Obstetrics \& Gynaecology, Aretaieion Hospital, National \& Kapodistrian University of Athens, Greece

2 2nd Academic Department of General Surgery, Aretaieion Hospital, National \& Kapodistrian University of Athens, Greece

* Corresponding author: 2nd Academic Department of General Surgery, Aretaieion Hospital, National \& Kapodistrian University of Athens, Greece, 76 Vas. Sofia Avenue, Athens, 11527, Greece; e-mail: arkoufan@gmail.com

Received: 25 October 2018

Accepted: 20 March 2019

Published online: 26 July 2019

Acta Medica (Hradec Králové) 2019; 62(2): 77-81

https://doi.org/10.14712/18059694.2019.107

(c) 2019 The Authors. This is an open-access article distributed under the terms of the Creative Commons Attribution License (http://creativecommons.org/licenses/by/4.0), which permits unrestricted use, distribution, and reproduction in any medium, provided the original author and source are credited. 


\section{INTRODUCTION}

In 1901, twenty-seven years after the original paper by Paget on this rare breast tumor, a French Dermatologist, Dubreuilh described the first case of Paget disease in the vulva (1). A few years earlier, in 1893, the first case of perianal Paget disease was reported (2). Extra-mammary Paget disease accounts for $10 \%$ of all cases and its estimated incidence is $0.7 / 100,000$ persons/year $(3,4)$. It affects more women than men and, in the majority of cases; it involves the vulva (85\%). Less than two hundred cases of Paget disease affecting the perianal area have been reported in the literature. When it is located in the vulvar and perineal area, invasive foci of adenocarcinoma may be found in $20-79 \%$ of cases. An invasive vulvar adenocarcinoma has been reported in $5-18 \%$ of cases $(5,6)$.

The disease in the vulvar and perineal area appears as thickened, erythematous and, often, ulcerative plaques of tissue, with no distinct borders. Under the microscope, a congregation of, mucin producing, intraepithelial neoplastic cells are evident. The pathogenesis of extramammary Paget disease remains unclear and three different theories have been proposed. The first theory suggests that Paget cells originate from mammary-like glands in the the interlabial sulci (7). The second theory suggests that they originate from multipotent stem cells in apocrine glands, found in the epidermal basal layer or infundibular stem cells of the hair follicles (8). The latest theory claims that the so-called Toker cells, with a single round nucleus and usually found in the areola and nipple of the breast, are the origin of extra-mammary Paget disease (9).

The cornerstone of extramammary Paget disease management is the wide local excision with lateral margins extending 2 to $3 \mathrm{~cm}$ beyond the affected areas (10). Often, skin or myocutaneous flaps are used for primary surgical closure. However, the disease can reoccur, even with negative surgical margins, especially when an underlying invasive adenocarcinoma is found. In the latter case, a more aggressive treatment is suggested with a concomitant inguino-femoral lymphadenectomy. Different adjuvant therapies have been proposed, i.e. radiotherapy, immuno-modulators, chemotherapy, or even, lately, target therapies, with various degrees of success.

\section{CASE HISTORY}

A 75-year old female patient presented with an erythematous plaque with white scaling, affecting the whole vulvar area and extending to the whole perineal and perianal area. She was complaining of itching, irritation and burning in the area. In the last five years, she had undergone, elsewhere, three wide local excisions of different areas of the vulva and perineum. The first local excision took place in 2007 , nine years earlier.

\section{CLINICAL EXAMINATION}

Neither the clitoris nor the labia were identified. Physical examination was very painful and, except for the erythematous plaques and scaling, several skin erosions were identified, especially, in the perianal area (Figure 1). On physical examination, no palpable inguino-femoral lymph nodes, nor hepato-splenomegaly were identified. On colposcopy, the disease seemed not to extend to the vaginal canal and the Papanicolaou test was negative. The patient underwent anoscopy and rectosigmoidoscopy, where no intraluminar lesions were identified, except for a solid nodule at the perianal area. The anal squamo-columnar junction appeared normal. Also, cystoscopy was normal, although the lesion seemed to affect the opening of the urethra. A computed tomography with intravenous radio-opaque material showed thickening of the vulvar area and perineum, but no enlarged inguino-femoral and pelvic lymph nodes.

The two previous wide local excisions had shown non-invasive Paget disease of the vulva with positive margins and, in the last one, the presence of invasive disease and two foci of underlying adenocarcinoma, up to a depth of $3.5 \mathrm{~mm}$. Positive margins for non-invasive Paget disease were found, even, in the last wide local excision. No lymphadenectomy was performed in any of the previous surgeries. The patient suffered from ischaemic cardiac disease and hypertension, for which she was under close observation and treatment by her cardiologist.

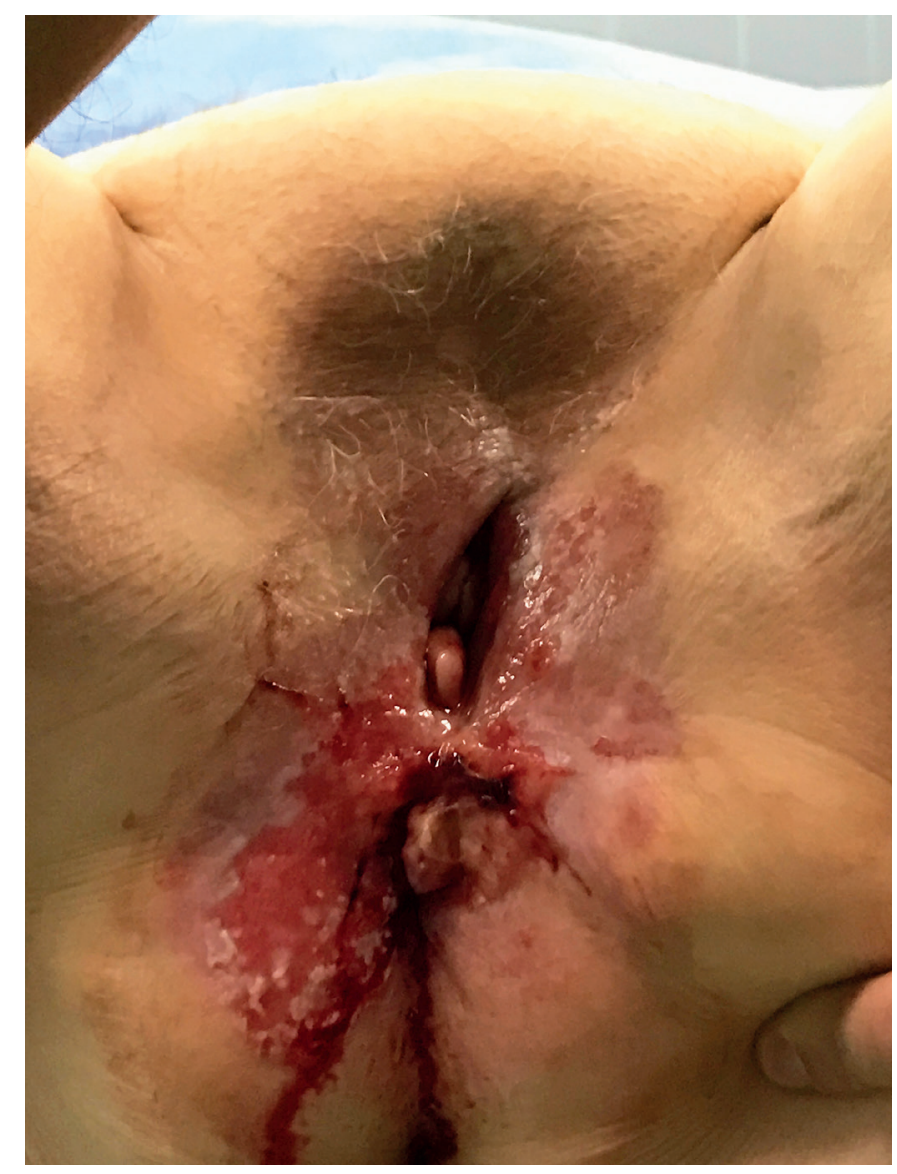

Fig. 1 Extent of disease before final surgery. 
Also, in the past, she had undergone a triple cardiac bypass operation.

\section{SURGICAL APPROACH \& PATHOLOGICAL FINDINGS}

In view of the above, and the negative CT findings for nodal disease, she was scheduled for a radical local excision of the lesion and reconstruction with no regional lymphadenectomy. In the operating theatre, a wide radical excision was performed, in order to include all visible lesions, with a $2 \mathrm{~cm}$ margin of healthy tissue. For this reason, the outer $1.5 \mathrm{~cm}$ of the urethra were mobilized and cut. The whole mons pubical area was mobilized and the transverse perineal muscles were exposed, along with the external anal sphincter. The whole lateral skin up to the underlying muscles and vaginal canal were mobilized (Figure 2).

Frozen sections confirmed the negative margins of excision, but the perineal nodule was reported as positive for high-grade adenocarcinoma. A further excision at the entrance of the anal canal confirmed the negative margins. Following mobilization of the adjacent skin and vagina, the pubic and the right lateral defects were closed on primary intention. For the closure of the left side, a Singapore flap was used. The flap was centered over the medial thigh crease. The flap measured $13 \times 5 \mathrm{~cm}$, with the long axis positioned parallel to the medial thigh crease.
The flaps were elevated in an anterior-to-posterior direction and included the deep fascia of the thigh adductor muscles. A posterior incision was extended through the skin and subcutaneous tissue to create an island flap, which was rotated approximately 90 degrees and passed through a tunnel, deep under the skin, lateral to where the labia majora were located in the past. Finally, a negative pressure drain was inserted and the flaps were sutured together to close the defect, with the apex of the flap sutured to the central gluteal area. There was no tension in any of the side of the flap (Figure 3 ). The blood loss was $200 \mathrm{cc}$ and the patient recovered well after the operation. Because of her medical history, she remained under close observation in the Intensive Care Unit. The wound was healing well, but, on the third post-operative day the patient complained of shortness of breath and chest palpitations. An ischaemic cardiac episode was diagnosed and the patient was transferred to the Cardiac Intensive Care Unit for observation. She recovered well, both, from the operation and the cardiac episode. The drain was removed on the 8th post-operative day, but a Foley urinary catherer was left in-situ for a total of 14 days, in view of the urethral cut and shortening. After removal of the Foley catheter, the patient was continent, both, for faeces and urine and the wound, including the flap, healed with no signs of infection, breakdown or haematoma. The patient was discharged from the hospital after 17 days. On final histology, a recurrence of non-invasive

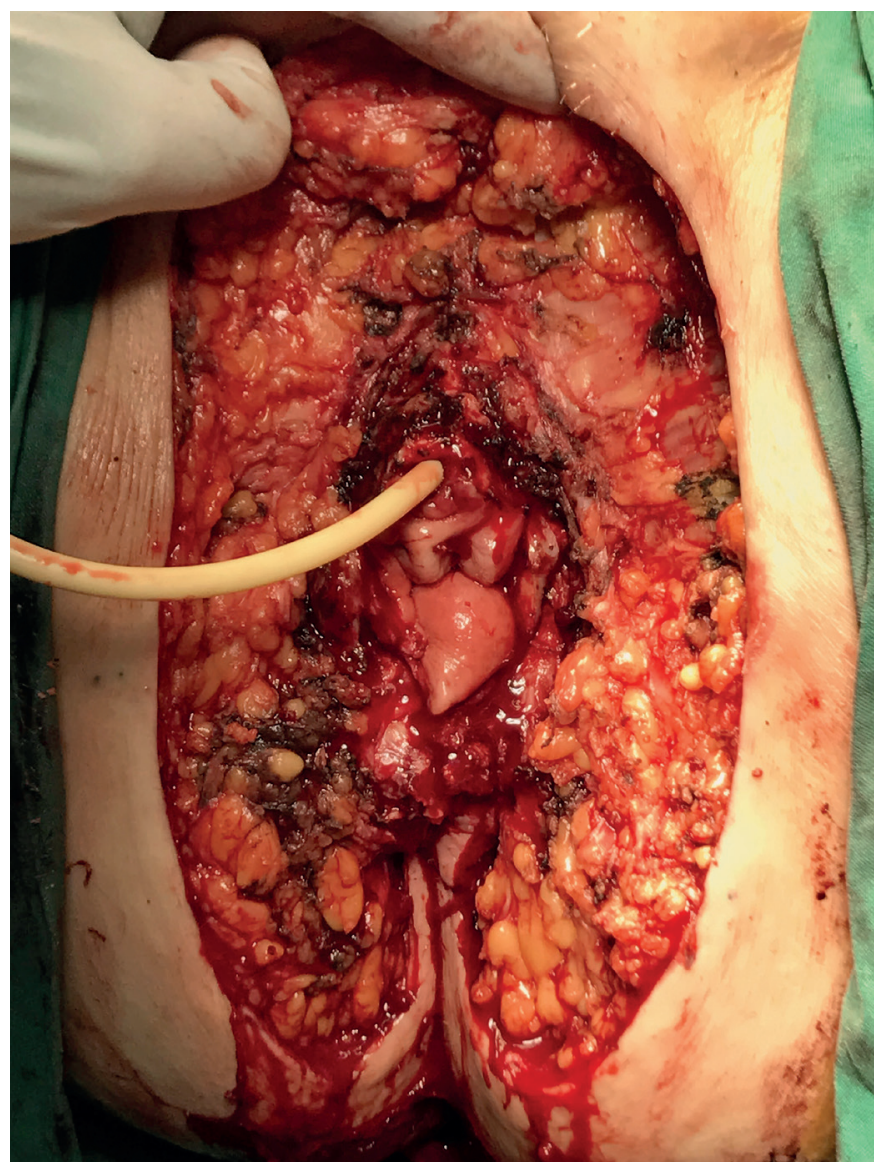

Fig. 2 Operative view after removal of lesion.

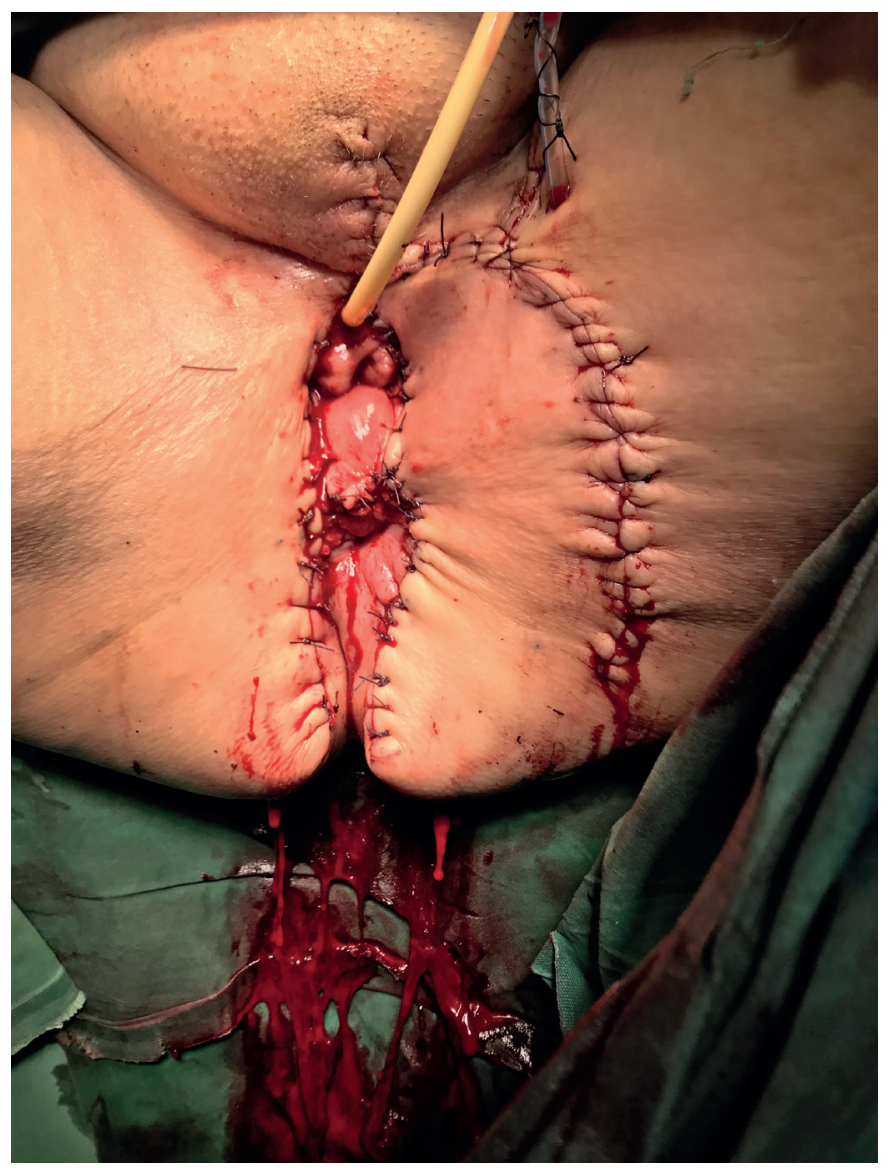

Fig. 3 Primary closure (Singapore flap on left side). 


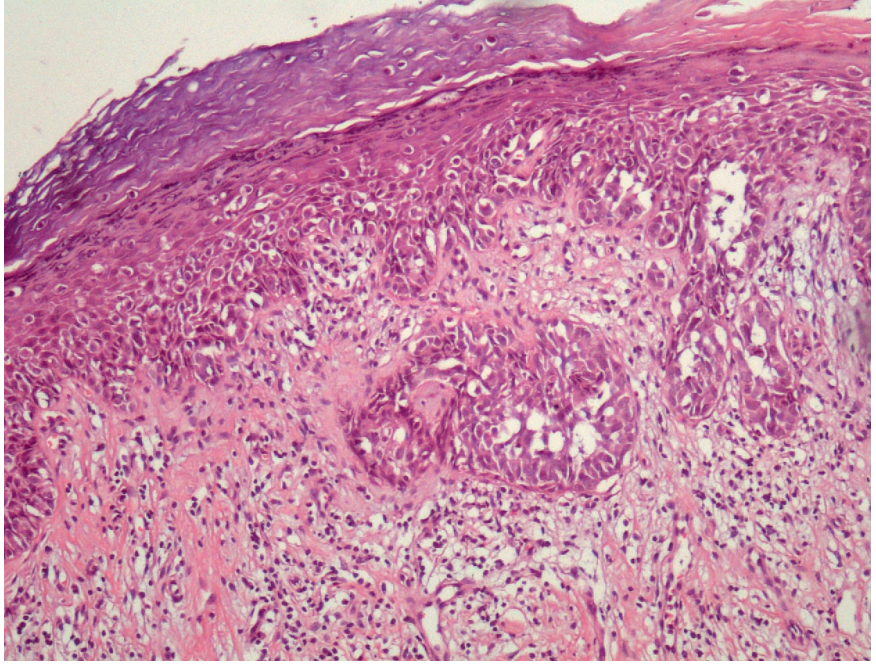

Fig. 4 Hyperkeratotic epidermis involved by Paget cells $(H+E \times 100)$.

Paget disease and a nodule of invasive high-grade adenocarcinoma were confirmed, along with the presence of multiple neoplastic vascular emboli. The disease extended close to the margins on the left lower side of the specimen, although there were many artefacts due to the use of electrocautery.

\section{OUTCOME}

Because of her complicated history, only local radiotherapy was proposed as adjuvant treatment. Eighteen months after completion of treatment, the patient remains well, with no signs of recurrence.

\section{DISCUSSION}

Extramammary Paget disease is a rare disease, mainly, affecting elderly postmenopausal women. Worldwide, there are only about 200 literature reports of perianal Paget disease. It is estimated that it represents, only, $1 \%$ of all malignant tumors in this area and 6.5\% of all Paget diseases (4, 5, 10). Based on its aetiological origin, EMPD is classified into primary, as in our patient's case, and secondary types. In the primary type, Paget's disease manifests as a (a) strictly intraepithelial disease, (b) intraepithelial disease with dermal invasion of Paget cells or (c) manifestation of an underlying adenocarcinoma of a skin appendage or subcutaneous gland. In the secondary type, the invasion is secondary to (a) an underlying anorectal adenocarcinoma, (b) an urothelial carcinoma or (c) an adenocarcinoma arising elsewhere (11).The clinical picture of the disease includes an erythematous or eczematous, poorly demarcated, plaque with white scaling and ulcerations. In most of the cases, the most commonly affected area is this of the labia majora, followed by the labia minora, clitoris, inguinal folds, the inner thighs and the perineal and perianal region. Conditions such as vulvovaginal candidiasis, eczema, psoriasis, lichen simplex chronicus and vulvar

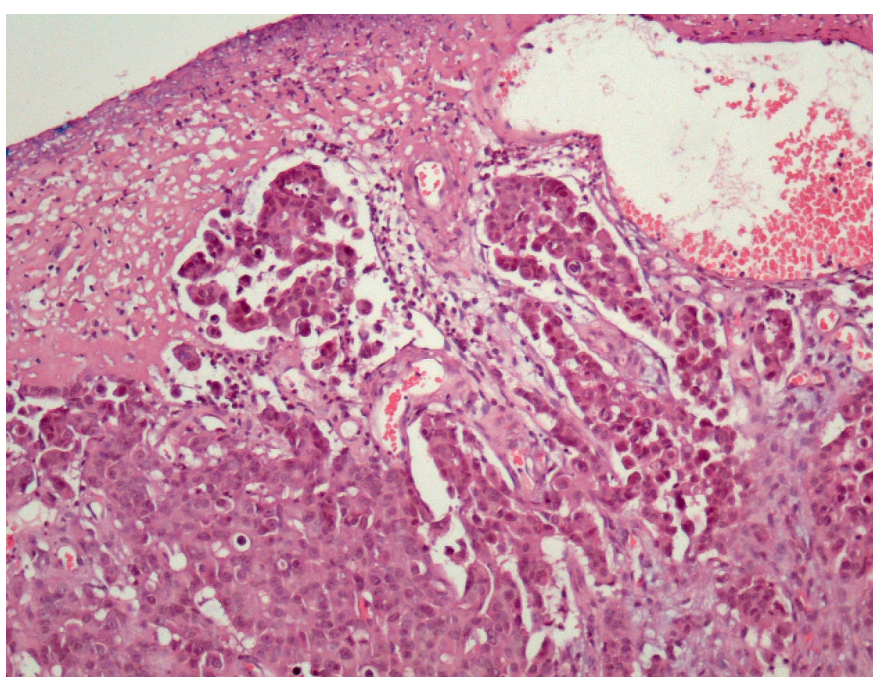

Fig. 5 Ulcerated, infiltrating, poorly differentiated adenocarcinoma $(\mathrm{H}+\mathrm{E} \times 200)$.

intraepithelial neoplasia (VIN) should be considered for the differential diagnosis of the vulvar and perineal Paget Disease (12). A detailed histological examination is of paramount importance in order to schedule the management of these patients. Especially, it is important to identify the presence of a co-existent invasive adenocarcinoma. It is identified in about one in four such patients $(24 \%)$ and it is associated with a high mortality rate, up to $46 \%$. Further, an underlying invasive adenocarcinoma necessitates more extensive surgery (inguino-femoral lymphadenectomy), which is indicated when there is invasive Paget disease of more than $1 \mathrm{~mm}$ in depth. The role of sentinel node biopsy is not yet clearly defined. The desirable free surgical margins range from 1 to $5 \mathrm{~cm}$. After surgical treatment of vulvar Paget disease, reported local recurrence rates vary from 34 to $56 \%$. Reconstructive and plastic surgery may be required in order to close the large skin defects. Radiotherapy, both as primary and adjuvant therapy with invasive adenocarcinoma, has been reported, with doses varying from 40 to $65 \mathrm{~Gy}$. Satisfactory results have been reported, with long-term recurrences of $<20 \%$. Also, recent data suggest that a dose of approximately 50Gy could eradicate microscopic disease near positive surgical margins and subclinical lymph node metastases $(19,20)$. The initial experience with Imiquimod shows impressive results in eradicating not only vulvar recurring disease, but, also, disease in the inguinal area. Further results are awaited, as regular, long treatment may produce severe side effects and discontinuation of it (18). Several chemotherapy regimes have been used, mainly for metastatic disease in extra- mammary Paget disease, with various degrees of clinical response. Currently, chemotherapy is, mainly, reserved for distant metastases and palliative treatment (21). Recently, chemotherapeutic agents, in combination with Trastuzumab (Herceptin), have been used for treating metastatic disease with success (22).

In summary, the invasive vulvo-perineal Paget disease requires aggressive surgery, involving radical removal of the vulva and perineum, along with an inguino-femoral lymphadenectomy. As the disease mainly affects women of 
advanced age, completeness of treatment may be compromised by several co-morbidities. Even so, local radiotherapy appears to be effective in eradicating microscopic local and lymph node disease. Currently, chemotherapy serves, mainly, as a palliative treatment modality for distant metastases, although its use in combination with Herceptin needs further investigation. Recently, Imiquimod, has produced impressive results in local and metastatic disease and further trials are underway.

\section{REFERENCES}

1. Dubreuilh W. Paget's disease of the vulva. Br J Dermatol 1901; 13: 407-13.

2. Darier J, Coulillaud P. Concerning a case of Paget disease of the perineal and scrotal area. Ann Dermatol Syphiligr 1893; 4: 25-33.

3. Fardal R, et al. Prognosis in cutaneous Paget's disease. Postgrad Med J 1964: 36: 584-93.

4. Kanitakis J. Mammary and extramammary Paget's disease. J Eur Acad Dermatol Venereol 2007; 21: 581-90.

5. Lloyd J, Flanagan AM. Mammary and extrammary Paget disease. J Clin Pathol 2000; 53: 742-9.

6. Karam A, Dorigo O. Increased risk and pattern of secondary malignancies in patients with invasive extramammary Paget disease. Br J Dermatol 2014; 170: 661-71.

7. van der Putte SC. Mammary-like glands of the vulva and their disorders. Int J Gynecol Pathol 1994; 13: 150-60.

8. Regauer S. Extramammary Paget's disease - a proliferation of adnexal origin? Histopathology 2006; 48: 723-9.

9. Willman J, Golitz L, Fitzpatrick J. Vulvar clear cells of toker. Am J Dermatopathol 2005; 27: 185-8.

10. Minicozzi A, Borsellino G, Momo R, Steccanella F, Pitoni F, de Manzoni G. Perianal Paget's disease: presentation of six cases and literature review. Int J Colorectal Dis 2010; 25: 1-7.
11. Wilkinson EJ, Brown HM. Vulvar Paget disease of urothelial origin: a report of three cases and a proposed classification of vulvar Paget disease. Hum Pathol 2002; 33: 549-54.

12. Terlou A, Blok LJ, Helmerhorst TJ, van Beurden M. Premalignant epithelial disorders of the vulva: squamous vulvar intraepithelial neoplasia, vulvar Paget's disease and melanoma in situ. Acta Obstet Gynecol Scand 2010; 89: 741-8.

13. Chanda JJ. Extramammary Paget's disease: prognosis and relationship to internal malignancy. J Am Acad Dermatol 1985; 13: 1009-14.

14. Ito Y, Igawa S, Ohishi Y, Uehara J, Yamamoto AI, Iizuka H. Prognostic indicators in 35 patients with extramammary Paget's disease. Dermatol Surg 2012; 38: 1938-44.

15. Kusatake K, Harada Y, Mizumoto K, et al. Usefulness of sentinel lymph node biopsy for the detection of metastasis in the early stage of extramammary Paget's disease. Eur J Dermatol 2015; 25: 156-61.

16. Nakamura Y, Ishitsuka Y, Nakamura Y, et al. Modified gluteal-fold flap for the reconstruction of vulvovaginal defects. Int J Dermatol 2010; 49: 1182-7.

17. Zhu Y, Ye DW, Chen ZW, Zhang SL, Qin XJ. Frozen section-guided wide local excision in the treatment of penoscrotal extramammary Paget's disease. BJU Int 2007; 100: 1282-7.

18. van der Linden M, Meeuwis KA, Bulten J, Bosse T, van Poelgeeste MI, de Hullu JA. Paget disease of the vulva. Crit Rev Oncol Hematol 2016; 101: 60-74.

19. Karam A, Dorigo O. Treatment outcomes in a large cohort of patients with invasive Extramammary Paget's disease. Gynecol Oncol 2012; 125: 346-51.

20. Hata M, Koike I, Wada H, et al. Radiation therapy for extramammary Paget's disease: treatment outcomes and prognostic factors. Ann Onc 2014; 25: 291-7.

21. Hatta N, Yamada T, Hirono T, et al. Extramammary Paget's disease: treatment, prognostic factors and outcome in 76 patients. Br J Dermatol 2008; 158: 313-8.

22. Ogawa T, Nagashima Y, Wada H, et al. Extramammary Paget's disease: analysis of growth signal pathway from the human epidermal growth factor receptor 2 protein. Hum Pathol 2005; 36: 1273-80. 